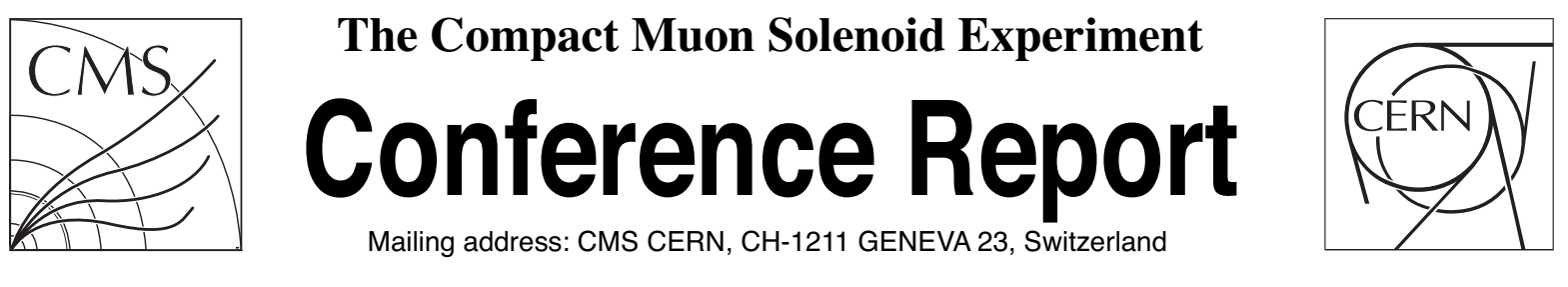

07 November 2017 (v3, 11 November 2017)

\title{
Offline Reconstruction Algorithms for the CMS High Granularity Calorimeter for HL-LHC
}

\author{
Z. Chen, C. Lange, E. Meschi, E. Scott and C. Seez \\ for the CMS Collaboration
}

\begin{abstract}
The upgraded High Luminosity LHC, after the third Long Shutdown (LS3), will provide an instantaneous luminosity of $7.5 \times 10^{34} \mathrm{~cm}^{-2} \mathrm{~s}^{-1}$ (levelled), at theCollaboration price of extreme pileup of up to 200 interactions per crossing. Such extreme pileup poses significant challenges, in particular for forward calorimetry. As part of its HL-LHC upgrade program, the CMS collaboration is designing a High Granularity Calorimeter to replace the existing endcap calorimeters. It features unprecedented transverse and longitudinal segmentation for both electromagnetic and hadronic compartments. The electromagnetic and a large fraction of the hadronic portions will be based on hexagonal silicon sensors of $0.5-1 \mathrm{~cm}^{2}$ cell size, with the remainder of the hadronic portion based on highly-segmented scintillators with SiPM readout. Offline clustering algorithms that make use of this extreme granularity require novel approaches to preserve the fine structure of showers and to be stable against pileup, while supporting the particle flow approach by enhancing pileup rejection and particle identification. We discuss the principle and performance of a set of clustering algorithms for the HGCAL based on techniques borrowed from machine learning and computer vision. These algorithms lend themselves particularly well to be deployed on GPUs. The features of the algorithm, as well as an analysis of the $\mathrm{CPU}$ requirements in the presence of large pileup, are presented.
\end{abstract}




\title{
Offline Reconstruction Algorithms for the CMS High Granularity Calorimeter for HL-LHC
}

\author{
Ziheng Chen, Clemens Lange, Emilio Meschi*, Edward Scott and Chris Seez \\ for the CMS Collaboration
}

\begin{abstract}
The upgraded High Luminosity LHC, after the third Long Shutdown (LS3), will provide an instantaneous luminosity of $7.5 \times 10^{34} \mathbf{c m}^{-2} \mathbf{s}^{-1}$ (levelled), at the price of extreme pileup of up to 200 interactions per crossing. Such extreme pileup poses significant challenges, in particular for forward calorimetry. As part of its HL-LHC upgrade program, the CMS collaboration is designing a High Granularity Calorimeter to replace the existing endcap calorimeters. It features unprecedented transverse and longitudinal segmentation for both electromagnetic and hadronic compartments. The electromagnetic and a large fraction of the hadronic portions will be based on hexagonal silicon sensors of $0.5-1 \mathrm{~cm}^{2}$ cell size, with the remainder of the hadronic portion based on highly-segmented scintillators with SiPM readout. Offline clustering algorithms that make use of this extreme granularity require novel approaches to preserve the fine structure of showers and to be stable against pileup, while supporting the particle flow approach by enhancing pileup rejection and particle identification. We discuss the principle and performance of a set of clustering algorithms for the HGCAL based on techniques borrowed from machine learning and computer vision. These algorithms lend themselves particularly well to be deployed on GPUs. The features of the algorithm, as well as an analysis of the CPU requirements in the presence of large pileup, are discussed.
\end{abstract}

Index Terms-LHC, CMS, Calorimetry, Reconstruction software, Clustering.

\section{INTRODUCTION}

$\mathbf{T}$ HE upgraded LHC, with its unprecedented instantaneous luminosity, will be a challenging environment for the forward calorimeter of CMS, both in terms of pileup interactions, and of integrated doses and neutron fluences. To address these challenges, CMS plans the replacement of the calorimeter system, in the pseudorapidity region $1.5<|\eta|<3.0$, with a new high-granularity sampling calorimeter (HGCAL), using silicon and scintillator as active material [1].

The HGCAL consists of an electromagnetic section of 28 layers of absorber and silicon sensors, with a depth of about $26 X_{0}$ and $1.6 \lambda$. The absorber is made up of $\mathrm{Pb}$ clad with stainless steel, $\mathrm{CuW}$ composite, and $\mathrm{Cu}$. A hadronic section follows, comprising 24 layers of stainless-steel absorbers totalling around $9.5 \lambda$. In the first 8 layers, silicon is again used as the detector; in the following 16 layers silicon is used in the high-fluence regions, whilst scintillating tiles readout by on-tile SiPMs are used in the low-fluence regions. Each of the 52 layers of the calorimeter are read out independently.

Z. Chen is with Northwestern University,

C. Lange and E. Meschi are with CERN

C. Seez and E. Scott are with Imperial College

* Corresponding author: emilio.meschi@cern.ch

\section{Clustering}

Clustering in the context of the HGCAL reconstruction is aimed at classifying energy measurements in the cells and assigning them to different physical objects. For a general purpose algorithm, this does not necessarily mean to identify the energy from a particle (e.g. a pion) as a single cluster. In fact, in most cases, the primary object identification relies on tracker data using particle-flow techniques [1]. The clustering only deals with grouping individual energy depositions (hits) belonging to the same shower.

The basic algorithm identifies the pattern of hits corresponding to a shower independently in each sampling plane. The set of 2-d clusters thus obtained is subsequently re-clustered in depth to group clusters from different layers into roads. The parameters of the road are used to initiate a candidate. Regression techniques can then be used to obtain the final parameters of a candidate reconstructed particle.

\section{A. Two Dimensional Clustering Based on Density}

The two-dimensional clustering algorithm is inspired, with some modification, to the one described in reference [2]. It starts by defining a local energy density of each hit cell $i$ in a layer as

$$
\rho_{i}=\sum_{j} \chi\left(d_{i j}-d_{c}\right)
$$

where $d_{i j}$ is the 2-d distance between the centers of cells $i$ and $j$, and $d_{c}$ is an arbitrary critical distance. The "kernel function" $\chi$ is defined as

$$
\chi\left(d_{i j}-d_{c}\right)= \begin{cases}E_{j} & \text { if } d_{i j}-d_{c}<0 \\ 0 & \text { otherwise }\end{cases}
$$

where $E_{j}$ is the energy of the $j^{\text {th }}$ calorimeter hit, $j$ enumerates hits on the given layer and takes all values including $j=i$. Basically, $\rho_{i}$ is the sum of the energy of all hits that are closer to hit $i$ than some cutoff distance $d_{c}$. The choice of this cutoff distance should not be overly important, but the stability of the algorithm for meaningful variations of $d_{c}$ must be studied in detail. The density map thus obtained is used to calculate, for each hit cell, the minimum distance to another hit with higher density

$$
\delta_{i}=\min _{j: \rho_{i}<\rho_{j}} d_{i j}
$$

Hits in a given layer are thus arranged in the $\delta$ - $\rho$ parameter space as shown in the example of Fig. 1. As can be seen in the figure, most of the hits are grouped in two categories: hits 

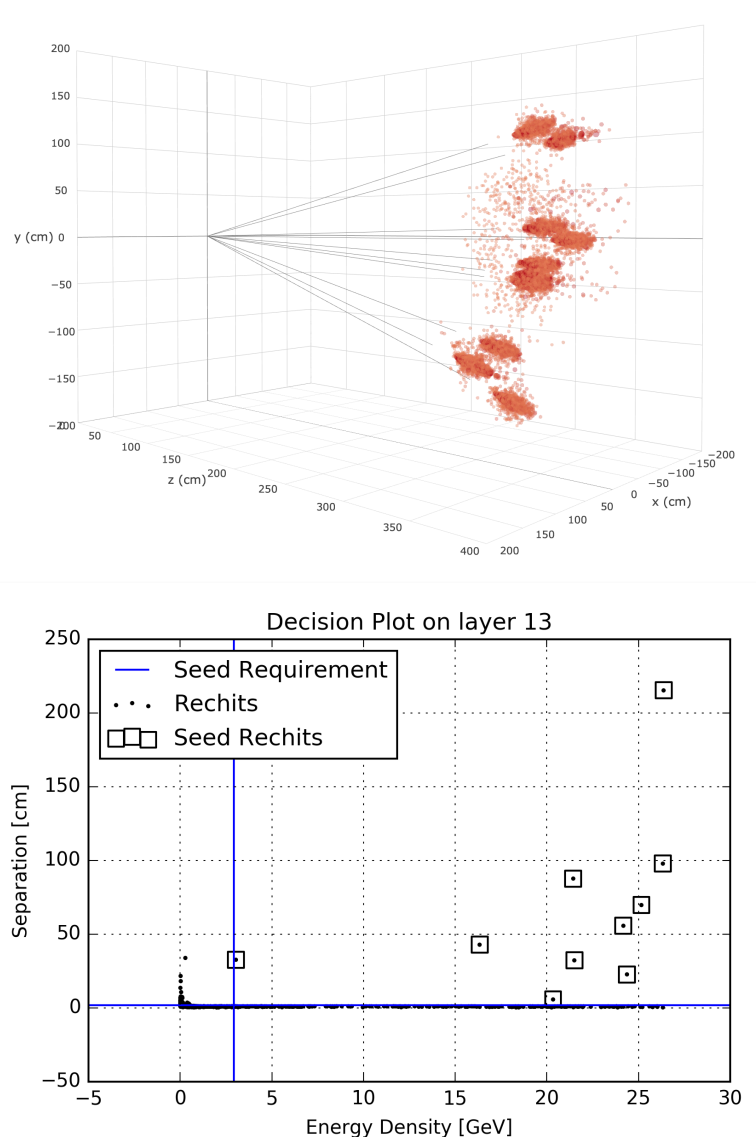

Fig. 1. Ten showers from photons of $P_{t}=35 \mathrm{GeV}$ (top). In the "separation" $(\delta)$ - energy density $(\rho)$ scatter plot (bottom) for layer 13 of the electromagnetic portion of HGCAL, each point represents an individual hit. Cluster centers (seeds) are easily recognisable as hits with large $\delta$ and relatively large $\rho$ (squares).

with large $\delta$ but small $\rho$ are isolated hits consistent with noise; hits with large $\rho$ but small $\delta$ form the core of a cluster (whose center is one of the hits above and to the right of the cuts).

Cluster centers are singled out as hits for which both $\delta$ and $\rho$ are large. For this purpose, the hits are ordered in decreasing $\delta_{i}$. All hits with $\delta_{i}>d_{c}$ are potential cluster centers if and only if their local density $\rho_{i}$ is larger than some value $\rho_{c}$, but in any case larger than some multiple of the noise threshold of the cell in question (easily recognisable in Fig. 1). Again, the dependence of results on the optimal value of $\rho_{c}$, which should be tuned to control the number of clusters while maintaining full efficiency, must be determined from the data.

Once the cluster centers have been identified, the list of hits ordered by decreasing values of $\rho_{i}$ is used to assign every hit to a cluster. The hit with the highest density in a layer is by definition the initiator of the first cluster. By assuming that a hit always belongs to the same cluster as its nearest neighbor with higher energy density, a single iteration over the ordered list of hits is sufficient to assign each and every hit above the noise threshold to one and only one cluster.

\section{B. Fast Nearest Neighbor Search}

The above technique to identify local energy maxima and group energy depositions into clusters lends itself well to be run on many-core architectures, e.g. GPUs, where parallelism can be exploited by processing individual layers on different cores. Furthermore, efficient and fast space-partitioning containers can be used for nearest-neighbor (NN) search. The search time for nearest neighbors using such containers typically scales as $n \log n$, where $n$ is the number of points, while the brute-force approach scales as $n^{2}$. As an example, Fig. 2 illustrates the timing for the density-based algorithm described in the previous sections, where the density calculation is implemented as a brute-force NN search, compared to an implementation using a kd-tree [3].

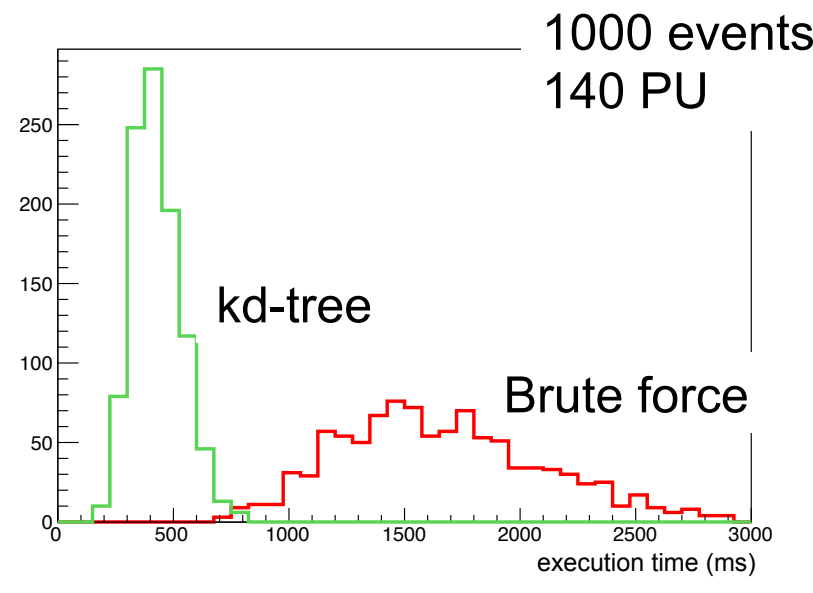

Fig. 2. CPU time to compute the hit density for 1000 events with 140 pileup interactions using brute force (red in online version) and kd-treebased approach (green in online version) to search for hit nearest-neighbors.

\section{Using 2-d Clusters}

Fig. 3 illustrates the performance of the 2-d-clustering on an event built by superimposing ten non-converting photons shot in different directions from a single point. It can be seen that 2-d cluster energy barycenter positions, defined as

$$
\vec{x}=\frac{\sum_{i \in c_{\ell}} \vec{x}_{i} E_{i}}{\sum_{i \in c_{\ell}} E_{i}}
$$

where the sum is over all hits $\mathrm{i}$ in each layer cluster $c_{\ell}$, align rather well along the individual shower axes.

In order to combine clusters from the individual layers into a possible shower candidate, we revert to a projective geometry using the pseudorapidity, $\eta$, and the azimuthal angle, $\phi$, of the 2-d cluster barycenters. Indeed, showers originating from objects produced near the interaction region will deposit energy at "nearly" constant $\eta-\phi$ positions. Fig. 4 exemplifies the kind of spatial accuracy to be expected in the determination of the shower axis: the $\phi$ and $\eta$ distributions of the 2-d cluster for non-converting $35 \mathrm{GeV} \mathrm{P}_{t}$ photons, shot in a fixed direction from the nominal detector center, are shown to have a width corresponding to a lateral accuracy of the order of few mm. After depth clustering, and assuming the primary 

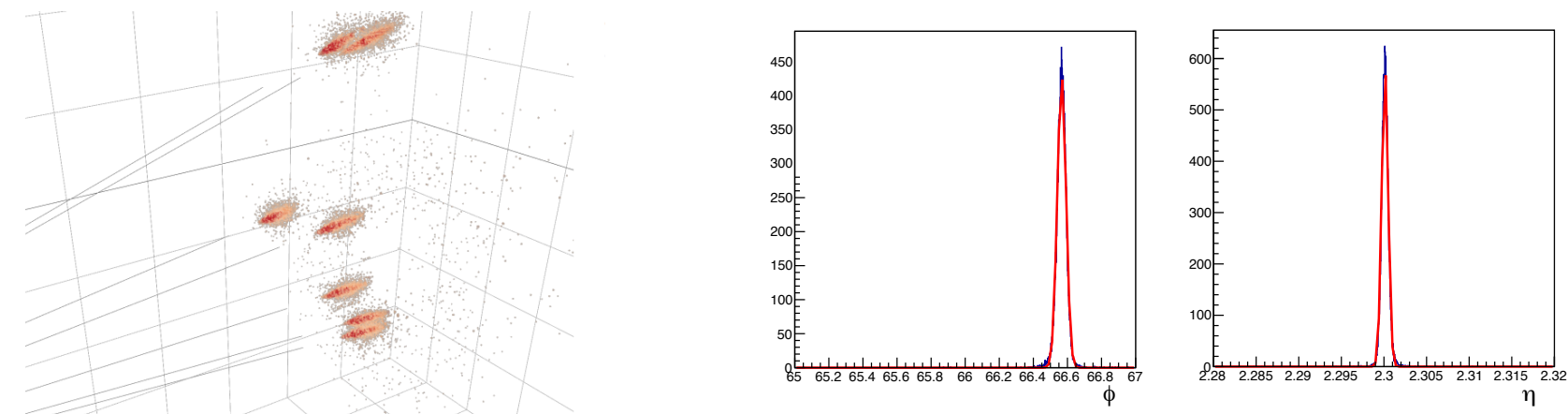

Fig. 4. $\phi$ (left) and $\eta$ (right) distributions of 2-d cluster barycenters for single non-converting photons shot at a fixed position. The mean values match the original position and the width corresponds to a position accuracy better than $3 \mathrm{~mm}$

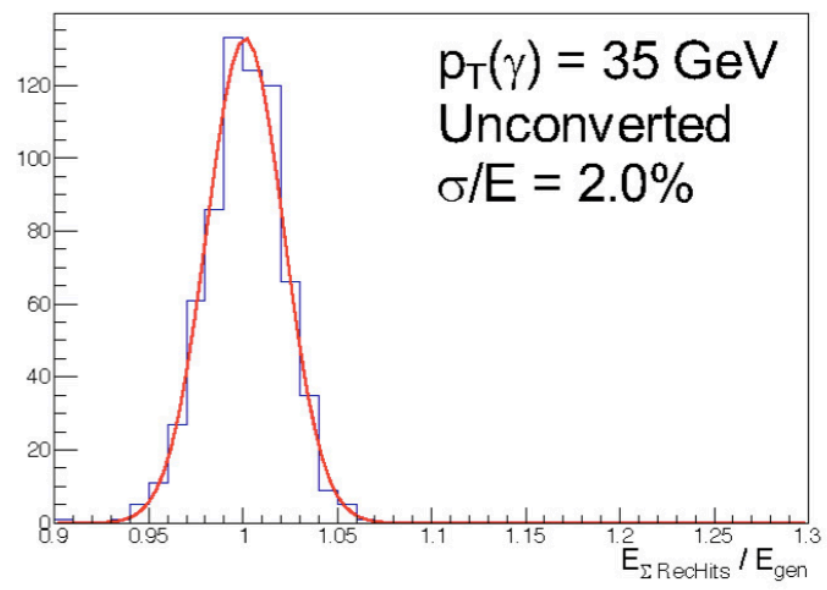

Fig. 5. Fraction of the original photon energy obtained by summing all energy depositions within $2 \mathrm{~cm}$ of the shower axis, identified as the line connecting the individual 2-d clusters in each layer (a calibration factor is applied to center the distribution at 1 ).

Fig. 3. The top picture shows the superposition on hits from showers of 10 non-converting photons. The bottom picture shows the coordinates of the 2-d cluster energy barycenters as found by the procedure outlined in the text.

vertex is known (for example from vertex reconstruction and primary vertex identification), the shower axis can be accurately determined by simply applying a linear regression to the individual 2-d-cluster barycenters.

As an illustration of the quality of the pattern recognition resulting from the two-step procedure outlined above, Fig. 5 shows the fraction of the original energy collected by summing all energy depositions within $2 \mathrm{~cm}$ of the estimated shower axis direction.

\section{Direct 3-D Density-Based Clustering}

A density-based algorithm in three dimensions, although less easy to parallelize, may be particularly interesting for the reconstruction of hadron showers. As can be seen from the example in Fig. 6, hadrons tend to produce showers consisting of segments which are loosely connected in depth.

Fig. 6. Artist rendition of the energy depositions from ten charged pion showers in the HGCAL. The size of the different "blobs" (and color in the online version) is proportional to the energy deposition in the corresponding layer. 
To ensure proper identification of the energy belonging to a shower initiated by a hadron, hit-by-hit depth correlation can be exploited, by introducing a "layer affinity". The 3-d density-based algorithm uses the hit coordinates $\left(x \prime, y^{\prime}, z^{\prime}\right)$, where $x /, y /$ are the central projection of a hit position onto the first layer, and $z \prime$ is the layer number times a constant $C$, which can be optimized to yield the best hit assignment. Fig. 7 and 8 illustrate graphically the preliminary results from a full 3-d implementation of the density algorithm for nonconverting photons and for pions, respectively.
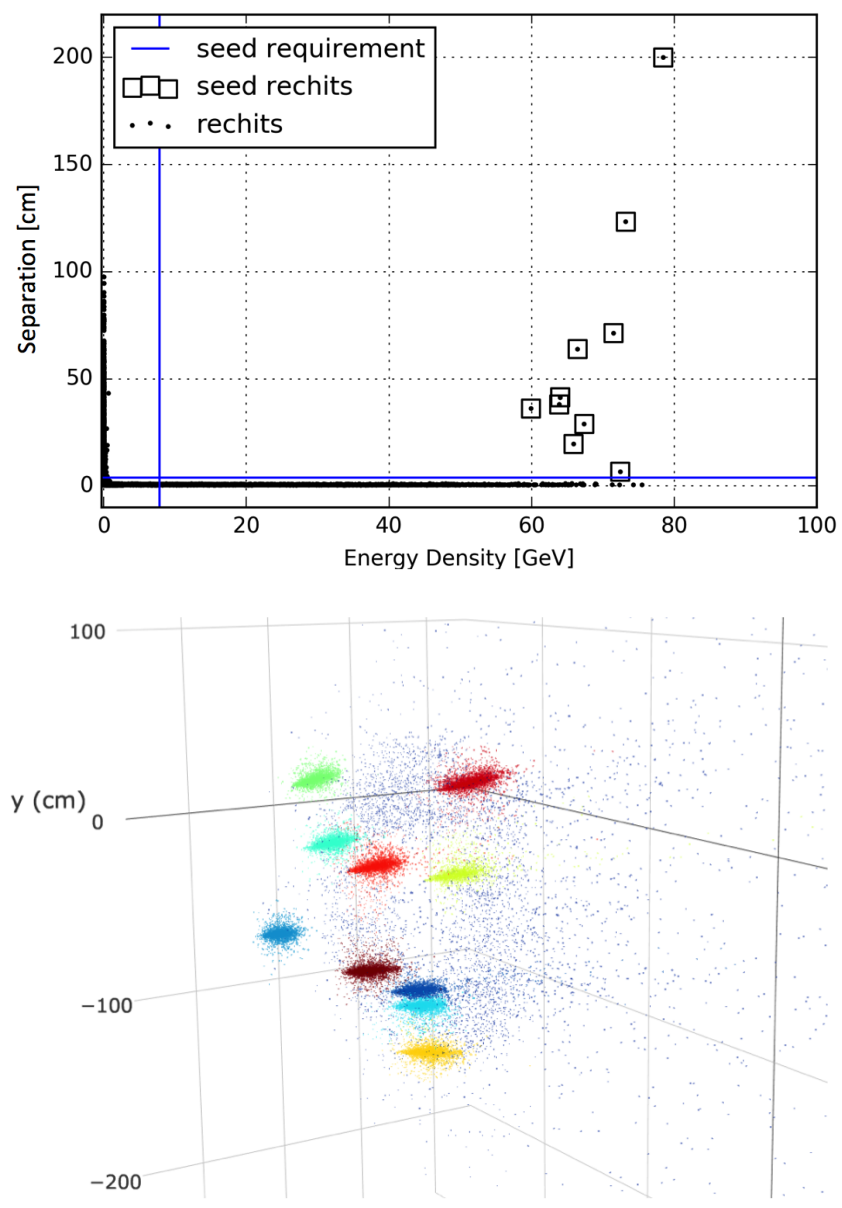

Fig. 7. Cluster center identification (top) using direct 3-d clustering for an event with ten non-converting photons; the hit assignment to different clusters is illustrated in the bottom plot by the different shades of grey (colors in the online version).

\section{CONCLUSION}

The HGCAL is a novel high-granularity calorimeter for the CMS Phase-2 upgrade, intended to instrument the region $1.5<|\eta|<3.0$. Its unprecedented granularity and optimal energy resolution are best adapted to work in the high multiplicity environment of HL-LHC and call for novel approaches to reconstruction.

A family of clustering algorithms based on energy density, inspired by classification techniques used in Machine Learning, promises optimal pattern recognition efficiencies. A
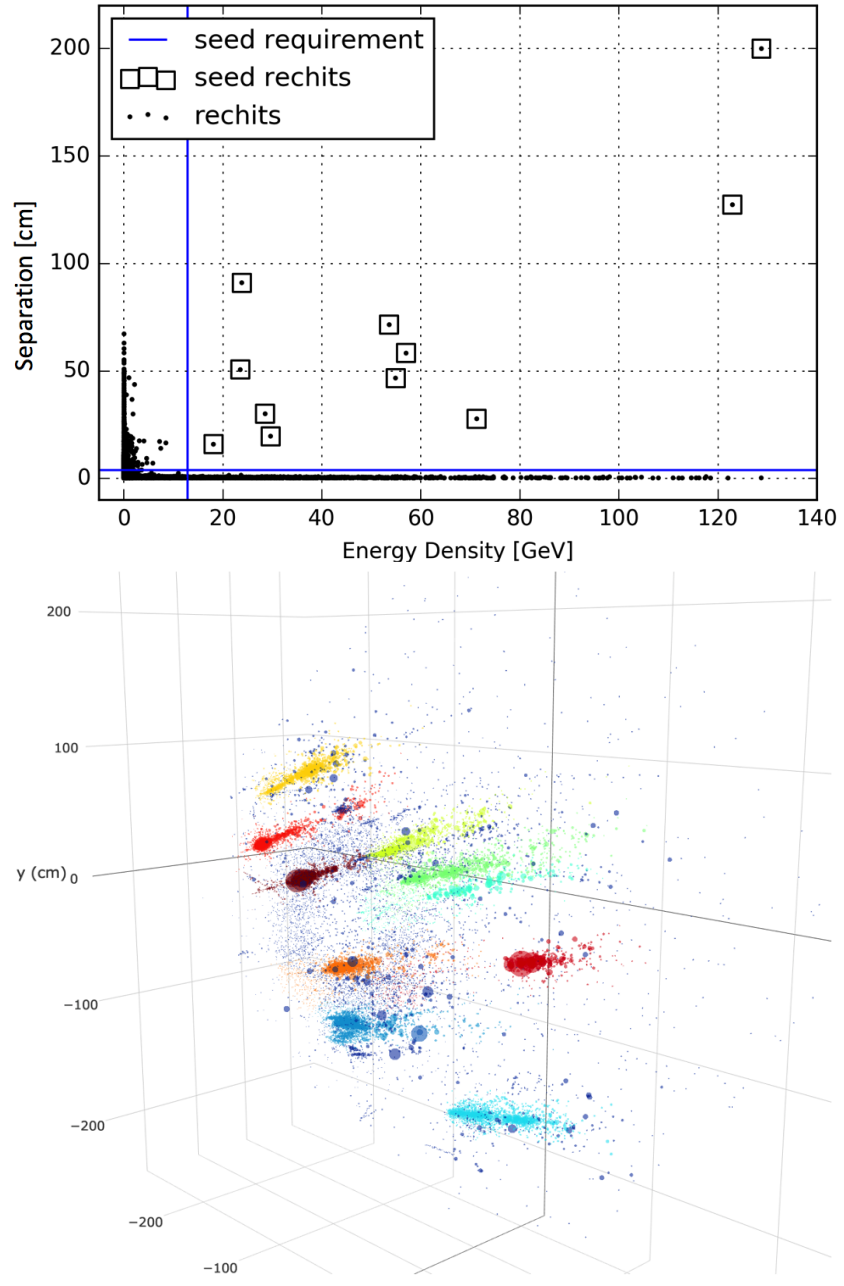

Fig. 8. Cluster center identification (top) using direct 3-d clustering for an event with ten charged pions; the hit assignment to different clusters is illustrated in the bottom plot by the different shades of grey (colors in the online version). As it is apparent from the plot, the 3-d algorithm is capable of identifying portions of the same shower that are disconnected in depth.

two-step approach, well suited for highly parallel processor architectures, gives promising results and can profit from using space-partitioning containers on GPUs to limit the computational cost.

A fully three dimensional version is also in the works, to best exploit hit-level depth correlation, which is particularly important for the efficient reconstruction of hadronic showers.

\section{REFERENCES}

[1] CMS Collaboration, Technical Proposal for the Phase-II Upgrade of the CMS Detector, CERN-LHCC-2015-010. LHCC-P-008. CMS-TDR-1502.

[2] Rodriguez, A and Laio, A, Clustering by fast search and find of density peaks, Science 344, 1961 (2014), 1492-1496.

[3] Bentley, J L, Multidimensional binary search trees used for associative searching, Communications of the ACM 18 (9). 